\title{
Elongated Cells May Unjam Cancers
}

\section{In tightly packed tissues, a cancer cell's motility is linked to the shape of the cell and of its nucleus.}

\section{By Marric Stephens}

C ancer cells in biological tissues sometimes behave like customers in a bar. At low densities, they are free to move around, but packed tightly, they jam together, inhibiting motion. Such density-dependent "jamming transitions" in tissues are well documented. Now, an experiment by Steffen Grosser, at Leipzig University, Germany, and colleagues supports the contested idea that jamming transitions in tumors can also be controlled by cell shape [1]. The results could provide a new way to predict metastasis risk.

For a tumor to grow and proliferate, cancer cells need to be able to move past one another. But cells in a tumor are typically packed at a density that should cause jamming and render the cancer cells immobile. Somehow, in a process that is still not completely understood, parts of the tumor can "fluidize."

To investigate this problem, Grosser and his colleagues examined "spheroids" - 3D cell cultures less than a millimeter across-derived from cancerous and healthy cell lines. The

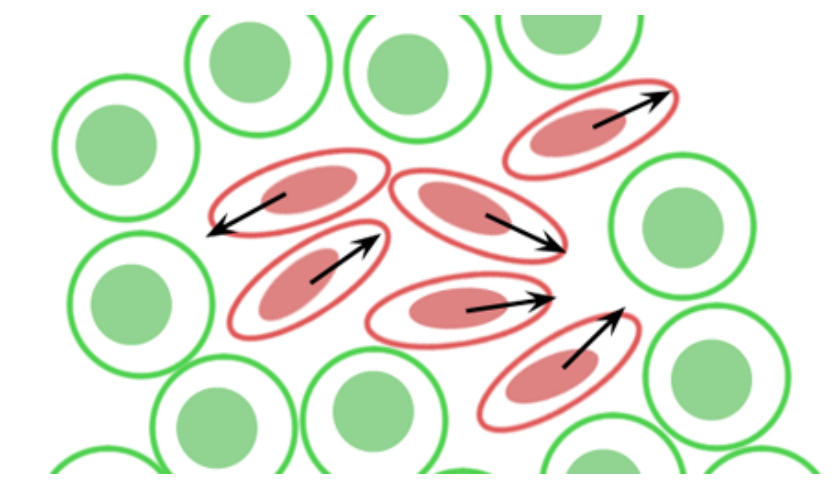

Credit: S. Grosser/Leipzig University team measured the mechanical properties of their spheroids by tracking each cell's motion as pairs of the spheroids merged together. Healthy spheroids behaved like solid balls with liquid outer layers, while cancerous spheroids coalesced like fully fluid droplets. In these fluid-like spheroids, the cells and their nuclei tended to be elongated, hinting at a shape-related fluidity control.

The researchers admit that this correlation between morphology and motility does not prove that the jamming transition is shape-controlled, and without analytical models, scientists will continue to argue whether shape or density is key. However, Josef Käs, who led the team, says that a shape control on motility seems intuitive. Even in a crowded bar, if you turn sideways, you might still squeeze through.

Marric Stephens is a Corresponding Editor for Physics based in Bristol, UK.

\section{REFERENCES}

1. S. Grosser et al., "Cell and nucleus shape as an indicator of tissue fluidity in carcinoma," Phys. Rev. X 11, 011033 (2021). 